\title{
Estrategia pedagógico-didáctica para promover la lectura crítica
}

\author{
LUIS PINCHAO BENAVIDES* \\ Universidad Mariana - Colombia \\ Recibido el 29-10-18; primera evaluación el 18-11-19; \\ segunda evaluación el 13-12-19; aceptado el 28-01-20
}

\section{RESUMEN}

El presente artículo recoge la experiencia vivida en la investigación de aula denominada: «La macroestructura textual como estrategia pedagógico-didáctica para promover la lectura crítica en estudiantes de pregrado». El proyecto, emprendido en enero de 2013 y finalizado en diciembre de 2014, asume uno de los problemas experimentados cotidianamente por los estudiantes en su devenir académico: la limitada capacidad de habitar en lectura crítica. Se trata de una investigación acción en educación que recurre a una ficha de lectura crítica, diseñada para identificar las macroestructuras semánticas en textos de corte argumentativos-descriptivos. Dicha actividad investigativa permitió observar paso a paso la manera cómo los estudiantes exploran y despliegan otras formas de lectura, distintas a la literal. El estudio ayudó a validar la ficha de lectura crítica como estrategia pedagógica-didáctica que favorece la lectura de alto nivel.

Palabras clave: lectura crítica, estrategia de aprendizaje, didáctica crítica, macroestructura semántica, textos argumentativos descriptivos.

\section{Pedagogical-Didactic Strategy to promote critical reading}

\section{Abstract}

This article gathers the experience of classroom research called: the textual macrostructure as a pedagogical-didactic strategy to promote critical reading in undergraduate students. The project undertaken in January 2013 and completed in December 2014, assumes one of the problems experienced daily by students in their academic development: the limited ability to live in critical reading. This is an

\footnotetext{
* Licenciado en Filosofía y Teología por la Universidad Mariana, Colombia. Magíster en Educación por la Pontificia Universidad Javeriana, Colombia. Magíster en Pedagogía por la Universidad Mariana. Docente-investigador, a tiempo completo, en la Universidad Mariana. Correo electrónico: Ipinchao@ gmail.com
} 
action research in education that uses a critical reading sheet, designed to identify semantic macrostructures in argumentative-descriptive texts. This research activity allowed us to observe step by step the way in which students explore and display other forms of reading, other than literal. The study helped validate the critical reading sheet as a pedagogical-didactic strategy that favors high-level reading.

Keywords: Critical reading, learning strategy, critical teaching, semantic macrostructure, descriptive argumentative texts.

\section{Estratégia pedagógica-didática para promover a leitura crítica}

\section{Resumo}

Este artigo reúne a experiência da pesquisa em sala de aula denominada: macroestrutura textual como estratégia pedagógico-didática para promover a leitura crítica em estudantes de graduação. $\mathrm{O}$ projeto realizado em janeiro de 2013 e concluído em dezembro de 2014, assume um dos problemas vivenciados diariamente pelos alunos em seu desenvolvimento acadêmico: a capacidade limitada de viver em leitura crítica. Trata-se de uma pesquisa-açáo em educação que utiliza uma folha de leitura crítica, projetada para identificar macroestruturas semânticas em textos argumentativo-descritivos. Essa atividade de pesquisa nos permitiu observar passo a passo a maneira pela qual os alunos exploram e exibem outras formas de leitura, além da literal. O estudo ajudou a validar a folha de leitura crítica como uma estratégia pedagógico-didática que favorece a leitura de alto nível.

Palavras-chave: Leitura crítica, estratégia de aprendizagem, ensino crítico, macroestrutura semântica, textos descritivos e argumentativos. 


\section{INTRODUCCIÓN}

El actual déficit en comprensión lectora y lectura crítica, experimentada a escala mundial, constituye un desafío para docentes y estudiantes; más aún cuando se cuenta con una generación de jóvenes un tanto cómodos y satisfechos en hacer lectura literal de las realidades que estudian, y muy pocas veces exploran otras dimensiones superiores de comprensión y evaluación. El problema se agrava cuando los docentes se desentienden de esta situación, y no generan escenarios para promover esta capacidad cognitiva en sus educandos. Aquí cobra pertinencia y valor la aventura pedagógica-investigativa, llevada a cabo en la Universidad Mariana, con estudiantes de pregrado, modalidad presencial, la cual buscaba desplegar la lectura crítica a partir de la identificación y construcción de la macroestructura textual.

Leer críticamente, en el ámbito universitario, no solo abre la mente y predispone a la persona al cambio, sino que concede autoridad moral para crear y recrear mundos posibles, aceptar o rechazar determinadas ofertas y demandas sociales. Según Girón, Jiménez y Lizcano (2008), la universidad coloca al estudiante en un escenario social distinto que le exige tomar posturas éticas y decisiones responsables, ante las realidades circundantes y los hechos que tiene que analizar, cuestionar y juzgar. Le permite vivir como ciudadano activo, crítico e intelectualmente perspicaz, capaz no solo de interpretar y analizar el mundo que le rodea, sino de adelantarse a los sucesos que están por acontecer.

El presente artículo recoge, en siete apartados, la experiencia investigativa denominada la «macroestructura textual como estrategia pedagógico-didáctica para promover lectura crítica en estudiantes de pregrado" (investigación de corte cualitativa, con enfoque crítico social). El primero se refiere a los antecedentes que dieron origen a la propuesta y a la pregunta problema, el segundo corresponde a una breve descripción de la intervención propuesta, el tercero alude a la revisión de la literatura, el cuarto da razón de los objetivos de la investigación, el quinto contempla aspectos inherentes al diseño de la investigación, tales como la metodología, muestra, instrumentos y una descripción paso a paso de la experiencia investigativa, el sexto recoge los resultados y los procesos de análisis. Finalmente, están las conclusiones y sugerencias emanadas del proceso investigativo. 


\section{MarCo teórico}

\subsection{Antecedentes que dieron origen a la propuesta y pregunta problema}

Paradójicamente hablando, en el mundo académico contemporáneo, especialmente en el ámbito universitario, el hábito lector tiende a perder el papel y el protagonismo que este juega en el acontecer cotidiano de la vida escolar. Un considerable grupo de educandos leen, pero de manera elemental, superficial y obligada, movidos más por el afán de aprobar que por el interés de aprender. Por su parte, el profesor espera que sus estudiantes no solo lean, sino que lleguen hasta la crítica de aquello que leen; más la realidad es otra, buena parte de los jóvenes universitarios no están en condiciones de realizar este tipo de exigencia académica.

En el ámbito universitario, el déficit en lectura crítica es cada vez más preocupante. Según Rugarcía (1999),

un buen número de estudiantes no comprende lo que saben, aducen teorías sin entender lo que ellas dicen, resuelven sin entender lo que resuelven, se encuentran anclados en una actitud pasiva frente a la vida, el estudio y la investigación (p. 66).

Este síndrome gana cada vez más adeptos y victimarios en todos los niveles de escolaridad, no obstante, donde más perjuicio ocasiona es en educación superior, pues el problema va más allá de tener bajo rendimiento académico, perder una asignatura o tener ínfimas calificaciones; dicha deficiencia cognitiva constituye un factor causante de desmotivación y deserción escolar, y un dispositivo de mediocridad profesional, corrupción y atraso social.

El interés por propiciar y promover lectura crítica en estudiantes universitarios está directamente relacionado con el componente ético de los futuros profesionales. La precaria comprensión y la complicidad de la actitud acrítica constituyen dos factores que ponen en riesgo la idónea preparación y desarrollo de las debidas habilidades intelectuales para analizar, interpretar y tomar decisiones de manera oportuna y correcta. La relación lectura crítica-universidad es tan cercana que la formación humana y profesional del egresado depende, en gran medida, de la calidad de lectura que se realice durante la estancia universitaria y fuera de ella.

Los factores comprometidos con la baja comprensión lectora y la incipiente lectura crítica de los estudiantes son diversos y están acompañados por la siguiente sintomatología: resistencia a la lectura de textos y documentos académicos, tendencia a querer aprender de manera rápida, fácil y sin mayor esfuerzo; 
actitud acrítica y contestataria frente a textos extensos y con vocabulario nuevo (los tildan de "filosóficos, incoherentes e impertinentes»). En consecuencia, para un buen porcentaje de jóvenes, su devenir académico transcurre al margen de la comprensión y la crítica; pasan por la universidad sin haber cuestionado aquello que estudian y viven la vida sin mayor reflexión, sin preguntarse seriamente sobre su propio porvenir y el de los demás.

El panorama anteriormente descrito convoca a aunar esfuerzos y medios apropiados para hacer frente a este fenómeno coartador del proceso formativo de los universitarios. A ello apostó la presente investigación, la cual partió del supuesto de que, al implementar deliberadamente ciertos recursos didácticos que exijan la identificación de macroestructuras textuales, se pueden activar y promover la comprensión lectora y la lectura crítica en los estudiantes del nivel universitario. Siguiendo este orden de ideas, el problema de investigación quedó formulado de la siguiente forma: ¿De qué manera la identificación y construcción de la macroestructura textual propicia la lectura crítica en estudiantes que cursan las asignaturas de Humanismo Cristiano y de Ética, durante el período académico enero-junio de 2013, en la Universidad Mariana?

\subsection{Investigaciones que orientaron la implementación de la propuesta}

A continuación, se mencionan, en la Tabla 1, las investigaciones que más contribuyeron a fundamentar y enriquecer el estudio, objeto del presente artículo. Todas, indistintamente de la comunidad de destino y del año en que fueron desplegadas, han planteado alternativas posibles para mejorar la comprensión lectora y ascender a la dimensión de la lectura crítica.

Tabla 1. Tesis relacionadas con la temática de la presente investigación

\begin{tabular}{llc}
\hline \multicolumn{1}{c}{ Investigación } & \multicolumn{1}{c}{ Autor } & Ańo \\
\hline $\begin{array}{l}\text { Bases para la comprensión organizativa } \\
\text { del texto }\end{array}$ & García Pilar, Elena & 2011 \\
\hline $\begin{array}{l}\text { La lectura crítica en los libros de texto de } \\
\text { educación secundaria }\end{array}$ & Zárate Pérez, Adolfo & 2010 \\
\hline $\begin{array}{l}\text { Conocimiento metacognitivo y } \\
\text { comprensión lectora }\end{array}$ & Villarroel Minerva, Rosas & 2010 \\
\hline $\begin{array}{l}\text { Características de comprensión lectora en } \\
\text { estudiantes universitarios }\end{array}$ & $\begin{array}{l}\text { Calderón Ibáńez, Arlenys y Quijano } \\
\text { Peńuela, Jorge }\end{array}$ & 2010 \\
\hline $\begin{array}{l}\text { La lectura crítica del artículo cientifico } \\
\text { como estrategia para el aprendizaje del } \\
\text { proceso de investigación }\end{array}$ & $\begin{array}{l}\text { Tarrés, María Cristina; Montenegro, } \\
\text { Silvana Marisa; D'ottavio, Alberto } \\
\text { Enrique y García Sánchez, Enrique }\end{array}$ & 2008 \\
\hline
\end{tabular}




\begin{tabular}{llc}
\hline \multicolumn{1}{c}{ Investigación } & \multicolumn{1}{c}{ Autor } & Año \\
\hline $\begin{array}{l}\text { Comprensión lectora de los textos } \\
\text { argumentativos en los niños de } \\
\text { poblaciones vulnerables escolarizados en } \\
\text { quinto grado educación básica primaria }\end{array}$ & Caballero Escorcia, Esmeralda Rocío & 2008 \\
\hline $\begin{array}{l}\text { Psicología de la comprensión textual y } \\
\text { control de la comprensión }\end{array}$ & Matéus Ferro, Geral Eduardo & 2007 \\
\hline $\begin{array}{l}\text { Aprender del texto: efectos de la estructura } \\
\text { textual y las estrategias del lector }\end{array}$ & McNamara, Danielle & \\
\hline $\begin{array}{l}\text { Comprensión lectora y funcionamiento } \\
\text { metacognitivo en estudiantes }\end{array}$ & Ochoa Angrino, Solanlly y Aragón & 2005 \\
universitarios & Espinosa, Lucero & \\
\hline $\begin{array}{l}\text { El uso de la estructura textual como } \\
\text { instrumento metodológico para mejorar }\end{array}$ & Lahuerta Martínez, Ana Cristina & 1996 \\
$\begin{array}{l}\text { la comprensión lectora de estudiantes de } \\
\text { inglés como lengua extranjera }\end{array}$ & & \\
\hline
\end{tabular}

Fuente: Elaboración propia a partir de esta investigación.

Por razones de extensión del artículo, se precisa únicamente el contenido de cuatro de las diez investigaciones mencionadas en la Tabla 1:

- La lectura crítica en los libros de texto de educación secundaria, llevada a cabo entre 2009 y 2010 por Pérez (trabajo final de su maestría en Lingüística y Aplicaciones Tecnológicas), el cual fue dirigido por Daniel Cassany. Esta investigación propone estudiar la concepción y la forma cómo abordan la lectura crítica los libros de texto de educación secundaria en el Perú, tanto los públicos como los privados. Se concluye que no existen diferencias significativas entre los libros de texto del Ministerio de Educación y las editoriales privadas Norma y Santillana sobre la concepción y el tratamiento metodológico de la lectura crítica.

- La lectura critica del artículo científico como estrategia para el aprendizaje del proceso de investigación, realizada por Tarrés, Montenegro, D’Ottavio y García (2008). Los tres primeros son catedráticos de la Universidad Nacional de Rosario (Argentina) y el último pertenece la Universidad de Salamanca (España). La propuesta se centró en promover la incorporación de competencias relacionadas con la generación del conocimiento científico. Los estudiantes lograron el aprendizaje de contenidos conceptuales y procedimentales, pero fallaron en los componentes de la lectura crítica. 
- Aprender del texto: efectos de la estructura textual y las estrategias del lector, proyecto de investigación realizado por McNamara (2004). Esta investigación aborda el problema de la comprensión lectora, observando los efectos de manipular la estructura del texto y de intervenir la estrategia lingüística, determinando cómo esos efectos dependen de las diferencias individuales, principalmente del conocimiento previo del estudiante. El objetivo fue encontrar soluciones reales para ayudar a los estudiantes a entender mejor los textos difíciles. La primera solución consiste en ofrecer al alumno textos relativamente cohesivos, estableciendo la mejor correlación posible entre lector y texto. Con ese fin, se crea un instrumento llamado CohMetrix, que evalúa la cohesión del texto y hace un cálculo estimativo de la coherencia del mismo basado en las aptitudes del lector. La segunda solución es entrenar a los alumnos en estrategias de lectura, centrada en la lectura activa del texto, tratando de explicarlo mientras se lee y realizando inferencias basadas en el texto $\mathrm{y}$ en el conocimiento previo para apoyar dichas explicaciones.

- El uso de la estructura textual como instrumento metodológico para mejorar la comprensión lectora de estudiantes de inglés como lengua extranjera, investigación realizada por Lahuerta (1996), financiada por la Universidad de Oviedo y publicada por la revista Aula Abierta en 1996. Analiza el efecto de la estructura textual (la organización retórica de un texto expositivo) en la lectura en inglés: la relación entre el uso de la estructura, por un lado; y la comprensión y la reproducción de información, por el otro. La investigadora concluye que la estructura resulta positiva para comprender la información de un texto; además encuentra que se da un cierto efecto entre la estructura y la reproducción de información. El estudio le permite hacer una clasificación entre lectores estratégicos, no estratégicos y decodificadores.

\section{Metodología}

\subsection{Objetivos de la investigación}

El objetivo general de la investigación en el aula fue analizar de qué manera la identificación de la macroestructura textual propicia la lectura crítica en estudiantes que cursan Humanismo Cristiano y Ética, durante el período académico enero-junio de 2013, en la Universidad Mariana. Los objetivos específicos fueron los siguientes: Identificar las dimensiones de lectura en la 
población objeto de estudio, a partir de un texto académico de corta extensión; aplicar una herramienta estratégica, basada en la macroestructura textual, para propiciar la lectura crítica en los cursos de Humanismo Cristiano y Ética; y, evaluar la efectividad de la herramienta estratégica en la consecución de la lectura crítica.

\subsection{Tipo de investigación}

La investigación se desplegó dentro del paradigma cualitativo, por cuanto su interés primordial es comprender las realidades para posibilitar mejores condiciones de vida y de desarrollo humano. Según Taylor y Bogdan (1986), el paradigma cualitativo es «aquel que produce datos descriptivos: las propias palabras de las personas, habladas o escritas, y la conducta observable» (p. 20). Se asume el enfoque crítico social, por cuanto se trata de una investigación acción de índole educativa que busca, por una parte, mejorar la condición lectora de los estudiantes, activar el pensamiento crítico, analítico y propositivo en ellos; y, por otra, mejorar la práctica pedagógica, hacer más efectiva la enseñanza y validar ciertos materiales didácticos. En los dos casos, se busca provocar el cambio conjuntamente con la comunidad escenario de la acción investigativa.

\subsection{Muestra}

La muestra estuvo constituida por 161 estudiantes: 52 estudiantes de ingeniería ambiental, segundo semestre; 30 de fisioterapia, tercer semestre; 31 del programa de contaduría pública, cuarto semestre; y 48 de enfermería, cuarto semestre del período académico enero-junio de 2013. Se consideraron los criterios de accesibilidad y disponibilidad sugeridos por John W. Creswell (2009); adicionalmente se establecieron otros dos criterios: dos grupos por investigador y que ostentasen un número de participantes que no fuera inferior a 20 ni superior a 60 .

\subsection{Instrumentos}

Los instrumentos que mediaron la actividad investigativa fueron la lista de chequeo, el diario de clase, la escala de valoración argumentada y la entrevista a grupo focal. La información suministrada por estos instrumentos fue procesada siguiendo las pautas del descubrimiento en progreso, sugerido por Taylor y Bogdan (2000), en los procesos de análisis de datos cualitativos. 


\subsection{Descripción}

Estos fueron los momentos vividos durante el proceso investigativo: El primer paso fue realizar un diagnóstico que permitiese establecer las dimensiones de lectura en que incursionan los estudiantes de ingeniería ambiental (III semestre), fisioterapia (II semestre), contaduría pública (IV semestre) y enfermería (IV semestre). Se efectuó en dos momentos: el primero, realizado en la primera sesión de aula y registrado en diario de clase; y el segundo, a través de una actividad académica de lectura analítica y crítica de un texto de corta extensión, cuyo producto fue valorado con la lista de chequeo como herramienta clasificatoria.

Durante el primer momento se propuso a los estudiantes estudiar la primera temática haciendo uso de la lectura pública dialogada, como estrategia de enseñanza-aprendizaje. En el caso del curso de Humanismo Cristiano, el tema estuvo relacionado con el concepto, origen y desarrollo del humanismo, y en el caso del curso de Ética, el tema fue de «alta» importancia y objeto de estudio de la ética. En términos generales, la actividad académica consistió en encomendar a cada estudiante la lectura en voz alta de un párrafo y expresar su parecer respecto a lo leído. La idea era provocar, no solo la participación en la actividad lectora, sino exigir a los estudiantes a expresar su postura personal, con el ánimo de percibir las posibles dimensiones de lecturas que estos eran capaces de producir; adicional a ello, la actividad académica solicitaba la escucha atenta, tanto del contenido del texto como de las alocuciones de los participantes, pues representaban puntos de partida para ascender a niveles de lectura de mayor complejidad.

El segundo momento del diagnóstico giró en torno a la lectura del texto correspondiente a la segunda temática a tratar en los cursos de Humanismo Cristiano y Ética, con el fin de hacer un ejercicio de lectura analítica y crítica. Se solicitó a los estudiantes la elaboración de un documento escrito que recogiera los puntos centrales abordados por el autor, y la adopción de una postura personal frente a los mismos. Tal actividad académica fue desarrollada bajo la modalidad de trabajo individual. El acompañamiento del profesor-investigador se limitó a precisar vocablos desconocidos o esclarecer dudas suscitadas por los estudiantes en el proceso lector y de producción escrita. Una vez recibido el producto solicitado, los investigadores recurrieron a la lista de chequeo, como herramienta clasificatoria, para identificar las dimensiones de lectura en que se encontraban los estudiantes y corroborar las percepciones obtenidas durante el desarrollo de la primera temática. Dicha técnica avista tres dimensiones de lectura: literal, inferencial y crítica; cada una de ellas contiene ítems, planteados a manera de criterios de verificación. 
El siguiente paso fue idear y aplicar una herramienta estratégica que propiciase la lectura crítica a partir de la identificación de las macroestructuras textuales. El reto consistió en diseñar un recurso conciso, claro y eficaz que permitiera a los estudiantes captar las macroproposiciones textuales, analizarlas, compararlas, confrontarlas y hacer juicios críticos sobre dichos contenidos. El resultado fue la ficha de lectura crítica, la cual fue sometida a prueba piloto y revisión de pares, antes de disponerla a los estudiantes para su respectivo estudio y aplicación. En la siguiente ilustración (Tabla 2), se puede apreciar el contenido general del instrumento aplicado a los textos elaborados por los estudiantes.

Una vez identificadas las deficiencias de lectura inferencial y crítica, vigente en los estudiantes adscritos a los cursos de Ética y Humanismo Cristiano, la tarea de los investigadores se centró en buscar posibles alternativas que permitieran propiciar ese tipo de lecturas. Idearon, entonces, una herramienta estratégica denominada ficha de lectura crítica, la cual invitaba al estudiante a desasir de la simple dimensión literal de leer las realidades y acceder a otras formas superiores de lectura. En términos generales, la ficha lectura crítica quedó constituida por cinco secciones: zona de identificación, características generales del texto, apartado proposicional, posturas de otros autores sobre el tema en cuestión y postura crítica frente a los contenidos del texto leído.

La zona de identificación de la herramienta pedagógico-didáctica solicita: número de consecutivo de la ficha, fecha de diligenciamiento de la misma, nombre de quien diligencia la ficha, título del artículo o tema a leer, autor de del tema, objeto de lectura y explicitación del propósito lector. La sección denominada características generales del texto hace alusión a los datos inherentes a la obra en general, la cual invita a considerar los siguientes puntos: tipo de texto (escrito, virtual, audiovisual, icónico), tipo de publicación (díptico, tríptico, folleto, periódico, libro, revista, web, video) y el registro de los datos del texto, según normas preferenciales o requeridas (APA, Icontec, MLA, Chicago, Vancouver).

El apartado proposicional está constituido básicamente por tres requerimientos, los cuales traen a colación la macroestructura semántica y las macroproposiciones textuales. Se deben redactar con las propias palabras o combinándolas con expresiones del texto original. Los tres requerimientos son los siguientes: tesis del autor (macroestructura semántica), ideas centrales (macroproposiciones), y el registro de las respectivas inquietudes y preguntas suscitadas durante el acto lector.

El componente denominado posturas de otros autores sobre el tema en cuestión corresponde a un espacio de confrontación y diálogo con otros 
autores que abordan la misma temática, aunque con otras perspectivas y planteamientos discursivos. Las posturas pueden ser a favor o en contra; la única condición es que tengan implicancia con el tema, objeto de comprensión y de crítica. Se sugiere que el acopio de tales versiones se describa, también, en forma proposicional.

La parte final de la ficha alude a la postura crítica del lector frente a los contenidos del texto leído. A manera de orientación, se plantean algunos interrogantes, tales como: ¿qué aceptas de lo leído y por qué?, ¿qué no compartes y por qué?, ¿qué bondades o amenazas devienen de los planteamientos del autor?, ¿qué piensas sobre la idoneidad del texto?, ¿qué valores humanos promueve?, ¿qué ideologías políticas subyacen en el texto? A continuación, se presenta el modelo de ficha de lectura crítica.

El siguiente paso del proceso investigativo fue llevar a escena la ficha de lectura. En términos generales se mencionan, a continuación, las principales apreciaciones y actitudes evidenciadas durante esta fase: Los estudiantes, por una parte, tienen dificultad a la hora de identificar y sintetizar el tema central y, por otra, no logran dimensionar la importancia de leer desde una intencionalidad determinada; un vasto número de estudiantes ha identificado y reconstruido las ideas claves de los textos sin explicitar un propósito claro de lectura; dan razón de lo leído, pero no saben, a ciencia cierta, diferenciar las ideas relevantes de aquellas que son mera explicación y refuerzo. En cuanto a la adopción de una postura crítica frente a los contenidos del documento leído, hay estudiantes que logran sorprender con sus perspectivas y argumentos, pero también existen quienes creen que una opinión personal es una crítica.

En total, se destinaron cinco sesiones de clase: cuatro para aplicar la ficha de lectura crítica con textos académicos bajo la tutoría del docente-investigador y una sesión sin acompańamiento con el fin de apreciar los alcances de la herramienta estratégica y evidenciar los beneficios de la misma en la consecución de la lectura crítica. Tal actividad se llevó a cabo con un grupo de 16 jóvenes, constituido por representantes de los cuatro programas académicos implicados en la investigación. Se efectuó en dos encuentros, en los cuales se aplicaron dos instrumentos respectivamente: Uno concerniente a la escala de valoración argumentada y el otro, a la entrevista a grupo focal.

Finalmente, se puso a disposición del grupo focal la ficha de lectura crítica para ser evaluada, según ítems y parámetros establecidos: «Muy en desacuerdo», «medianamente de acuerdo" y "muy de acuerdo». En la Figura 1, se puede apreciar el consolidado general de dicha evaluación. 
Tabla 2. Modelo de ficha de lectura crítica

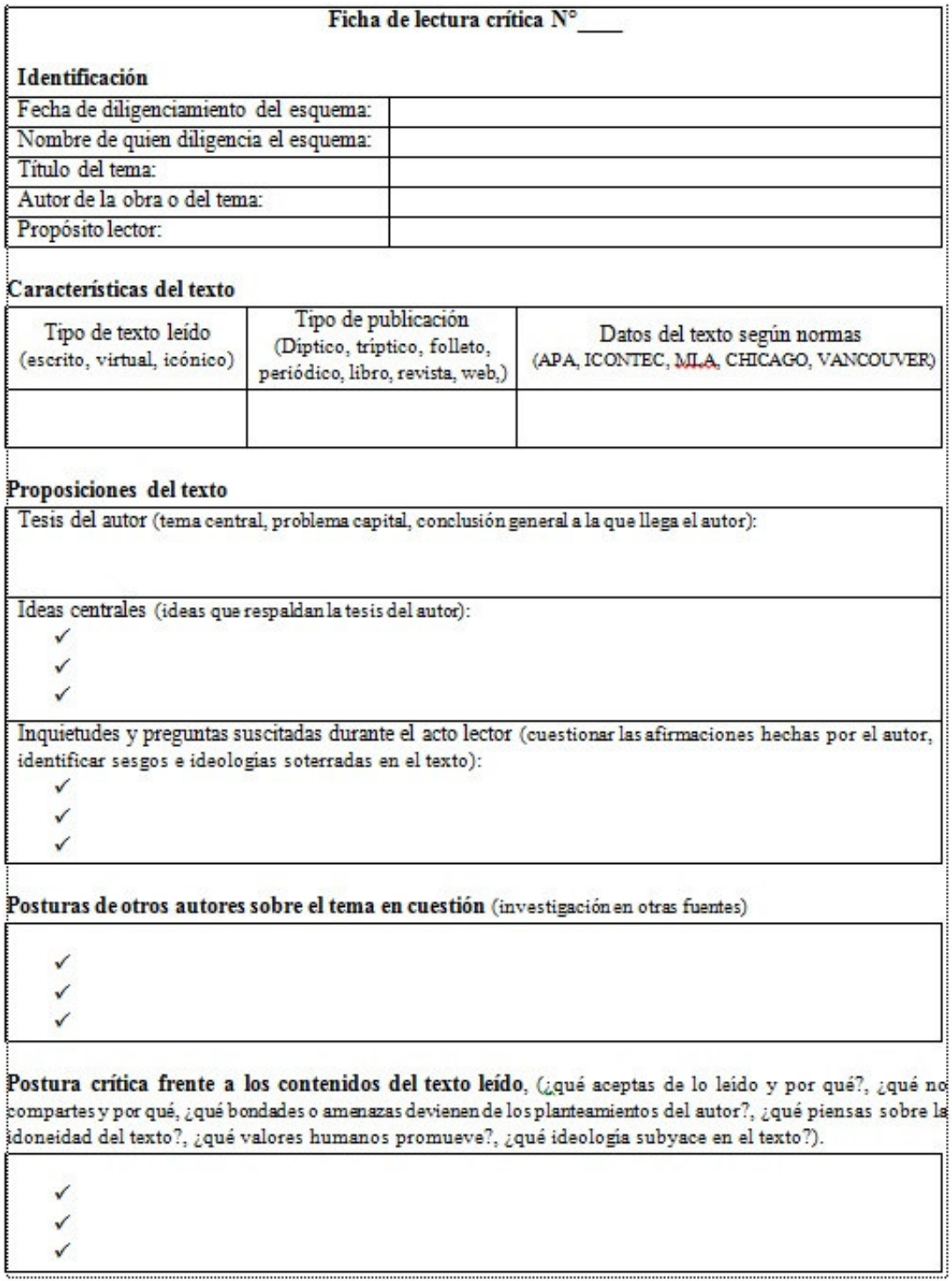

Fuente: Elaboración propia a partir de esta investigación. 


\section{Resultados}

Los resultados del diagnóstico se pueden apreciar en la siguiente ilustración:

Figura 1. Consolidación final del diagnóstico

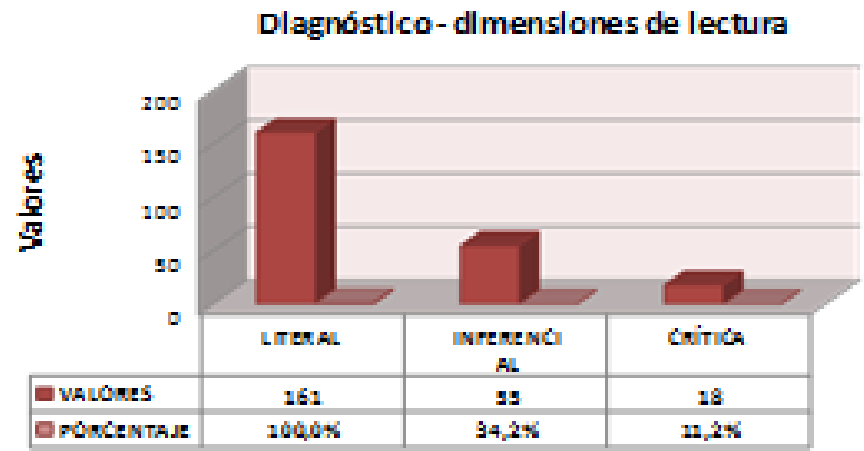

Fuente: Elaboración propia a partir de esta investigación.

Siguiendo los lineamientos de Taylor y Bogdan (1986), respecto a las etapas diferenciadas en los procesos de análisis de datos cualitativos, se llevó a cabo esta labor a través del descubrimiento en progreso que, según los autores mencionados, consiste en identificar temas y elaborar proposiciones teóricas. En consecuencia, siguieron las pistas temáticas concernientes a las dimensiones de lectura literal, inferencial y crítica, percibidas en los estudiantes y registradas en los diarios de clase de los docentes investigadores. Buscando temas emergentes y tipologías, se establecieron proposiciones agrupadas y proposiciones síntesis, asumidas como peanas para una eventual interpretación y discusión epistémica.

Como se puede observar en la Figura 2, es bastante limitado el número de educandos que se mueven en la dimensión de lectura crítica; sin embargo, un buen número de estudiantes muestra capacidad de llevar a cabo lectura analítica, interpretativa, deductiva, hipotética e implícita que, si bien es propia de la dimensión inferencial, representa un importante paso hacia la lectura crítica propiamente dicha. El número de estudiantes en esta dimensión de lectura es bastante precario en proporción con la población estudiantil objeto de diagnóstico. Pero, el suceso singular que atrapa la atención tiene que ver con el grado de escolaridad de los estudiantes que constituyen la muestra: Las tres cuartas partes se encuentran en tercer y cuarto semestre; nivel universitario que debería revelar una capacidad lectora de orden superior; sin embargo, los 
datos suministrados en la Figura 1 muestran que los estudiantes, a pesar de encontrarse casi a la mitad del tiempo previsto para finalizar su carrera profesional, se hallan aún anclados a formas elementales de lectura.

Asimismo, de los resultados sustraídos de los diarios de clase, levantados durante la etapa de la aplicación de la ficha de la lectura crítica, se pueden precisar de la siguiente manera. En primer lugar, los estudiantes tienen dificultad a la hora de identificar y sintetizar el tema central; creen que, si expresan la macroestructura textual o las macroproposiciones con sus propias palabras no van a ser valoradas, ni consideradas válidas por el profesor al momento de calificar, razón por la cual arguyen que prefieren tomar literalmente las expresiones del autor como estrategia para asegurar la aceptación y buena calificación. Por otro lado, gran parte de la población estudiantil da razón de lo leído, pero no sabe diferenciar las ideas relevantes de aquellas que son mera explicación y refuerzo.

Sobre adoptar una postura crítica frente a los contenidos del documento leído, es importante enfatizar nuevamente que existen estudiantes que consideran la opinión personal como sinónimo de crítica. Incluso, muchos de ellos se sienten competentes en lectura crítica al anteponer a sus intervenciones las expresiones "no estoy de acuerdo» o "no comparto lo dicho por el autor», desprovisto de argumentos y razonamientos claros.

Por grado de recurrencia, los diarios de clase, permiten inferir que la incursión de los estudiantes en las dimensiones de lectura mencionadas, especialmente en la inferencial y crítica, no es espontánea en ellos. Para conseguir tal efecto, el profesor se ve abocado constantemente a interpelar e inducir al educando para que este pueda analizar y ver realidades más allá de las que aparecen a primera vista, evaluar los planteamientos del autor desde criterios de relevancia e irrelevancia, entre otros. No se desconoce las capacidades y habilidades intelectuales de algunos estudiantes que hacen auténtica lectura crítica, pero su número es muy limitado, muy pocos alcanzan el análisis inferencial, y solo una minoría logra acceder a los terrenos de la lectura crítica, propiamente dicha.

Una vez finalizado el período de capacitación, entrenamiento, aplicación y socialización de la experiencia vivida por los estudiantes con la ficha de lectura crítica, llegó el momento de verificar los alcances de esta herramienta.

Como se puede apreciar más adelante, en la Tabla 3, el instrumento correspondiente a la escala de valoración argumentada contempló diez ítems que los estudiantes debían valuar siguiendo los parámetros de "muy en desacuerdo", «medianamente de acuerdo» $\mathrm{y}$ «muy de acuerdo». Además de optar por uno de los tres criterios, estaba el requerimiento de respaldar la opción con una breve 
y contundente justificación. A continuación, el resultado de esta actividad será explicada.

Los dieciséis (16) estudiantes que conforman el grupo focal encuentran que considerar el tema, el autor y el propósito de lectura es de capital importancia a la hora de adoptar una postura personal frente a lo leído, porque permite evaluar los planteamientos de un autor e identificar los puntos centrales que este aborda. Asimismo, creen que la acción de registrar preguntas suscitadas durante el proceso lector favorece la comprensión e incide en la calidad de los argumentos que se emiten respecto los puntos tratados en el documento, objeto de lectura. Algunos estudiantes manifiestan la conveniencia de colocar por escrito los interrogantes provenientes del acto lector, en tanto que ayuda a disipar dudas, inconsistencias, cegueras cognoscitivas, entre otras; al llevar los interrogantes al aula no solo se aclaran las situaciones confusas y se evitan falsas interpretaciones, sino que enriquece la cosmovisión del lector, equipándolo de argumentos contundentes e intervenciones coherentes respecto de los contenidos del texto leído.

Trece de quince estudiantes, exteriorizan que la ficha de lectura crítica invita al lector a confrontar el texto leído con otros autores que trabajan el asunto en cuestión. Esta actividad, según los estudiantes, permite comprender mejor el tema y sacar las propias conclusiones, lleva a conocer otras formas de pensar sobre un mismo tema y sirve para contemplar otros puntos de vista a favor o en contra. Ello se verá reflejado en la calidad y pertinencia de las respectivas posturas críticas, en tanto, no solo ayuda a ampliar la comprensión, sino a tener otros referentes para respaldar las posturas personales. Finalmente, consideran que esta iniciativa es una importante alternativa para no quedarse encasillado con la versión de un solo autor.

De igual manera, declaran que la posibilidad de confrontar el texto leído con otros autores corresponde a una actitud ética y, probablemente, esperada por el gestor de la obra. No obstante, dos de ellos están medianamente de acuerdo con este requerimiento de la ficha, porque no comparten la idea de tener que buscar, por cuenta propia, autores y teorías para confrontar el texto leído. Asimismo, un joven educando aduce estar completamente en desacuerdo con esta exigencia de la ficha, puesto que, para él, lo que realmente debe interesar es conocer únicamente el punto de vista del autor y emitir juicios sobre sus planteamientos.

Los dieciséis educandos que constituyen el grupo focal coinciden en manifestar que no se puede hacer lectura crítica si previamente no se identifican la tesis y las ideas centrales trabajadas por el autor. Uno de los estudiantes considera que se deben contemplar, también, las ideas secundarias por cuanto 
estas brindan elementos y detalles particulares a la hora de discutir lo esencial, contribuyendo significativamente en la percepción del sentido profundo del texto. Es importante resaltar el valor que le dan los estudiantes a la macroestructura textual, pues en sentido estricto la comprensión y la crítica es consecuencia directa de la capacidad humana para captar lo esencial.

Catorce de dieciséis estudiantes reconocen que la ficha de lectura crítica ayuda a reconocer la propiedad intelectual desde el mismo momento que invita a considerar al autor del texto, objeto de lectura; suceso que se concretiza en la sección de referenciación bibliográfica de la obra. Dos de ellos consideran este aspecto relativamente relevante, aduciendo que lo verdaderamente importante es la comprensión del texto, no la verificación de quién es el autor y demás datos que solo sirven como requisito formal. Si bien la ficha de lectura crítica representa un importante recurso garante del derecho de autoría, esta tarea reclama mayor acompańamiento por parte del docente, pues con mucha frecuencia los estudiantes no solo incurren en plagio, sino que ostentan una actitud indiferente frente a este tema.

Los dieciséis estudiantes, evaluadores de la ficha, aseguran que esta herramienta dinamizadora del aprendizaje permite entender, comprender, estructurar ideas y obtener aspectos claves de los textos; ayuda a tener más claro lo que el autor quiere dar a entender, a la vez, que propicia la lectura crítica y predispone al educando hacia la investigación, profundización, y discusión del tema propuesto. Asimismo, valoran las pistas orientadoras que comporta la ficha, en tanto, ayudan a generar lectura crítica. Exaltan de la ficha los siguientes aspectos: Motiva a encontrar bondades o amenazas, ayuda aceptar o rechazar con argumentos y facilita descubrir ideologías implícitas. Consideran que ello constituye una forma de encaminar a los estudiantes a la profundización de contenidos y a emprender procesos de investigación y reflexión.

Desde la perspectiva de los estudiantes entrevistados, la lectura crítica se concibe como la capacidad humana para evaluar los sucesos de la vida cotidiana y las obras de los autores. Se trata de procesos mediante los cuales se analiza y se interpreta con madurez y detalle. También, los estudiantes ratifican que, para realizar lectura comprensiva y crítica, se debe identificar necesariamente la tesis y las ideas centrales; aducen que, sin esta acción, todo intento por analizar y evaluar los contenidos de un determinado texto sería impertinente y poco ético. En este sentido, la ficha de lectura crítica cumple con la expectativa por la cual fue creada: Ayudar a captar los planteamientos esenciales de los textos expresados a través de macroproposiciones. 
Finalmente, los estudiantes coinciden en afirmar que todos los aspectos de la ficha de lectura crítica son fundamentales para lograr prácticas de lectura de orden superior; puntualizan que entre los talantes más relevantes y decisivos para su consecución están, además de la conveniente identificación de la tesis y las ideas principales, la formulación de preguntas personales suscitadas durante el acto lector y la confrontación del tema desde la perspectiva de otros autores.

Tabla 3. Consolidado-escala de valoración argumentada (evaluación Ficha de lectura crítica)

\begin{tabular}{|c|c|c|c|c|}
\hline $\mathrm{N}^{\circ}$ & Aspectos & $\begin{array}{c}\text { Muy en } \\
\text { desacuerdo }\end{array}$ & $\begin{array}{l}\text { Medianamente } \\
\text { de acuerdo }\end{array}$ & $\begin{array}{l}\text { Muy de } \\
\text { acuerdo }\end{array}$ \\
\hline 1 & $\begin{array}{l}\text { Deliberar sobre el tema, el autor y el } \\
\text { propósito de lectura constituye un } \\
\text { importante paso para quien desea hacer } \\
\text { lectura crítica. }\end{array}$ & & & 16 \\
\hline 2 & $\begin{array}{l}\text { Registrar preguntas que surgen durante la } \\
\text { lectura, constituyen importantes puntos } \\
\text { de referencia al momento de emitir juicios } \\
\text { críticos sobre los contenidos de un texto. }\end{array}$ & & & 16 \\
\hline 3 & $\begin{array}{l}\text { La ficha permite confrontar las afirmaciones } \\
\text { del autor con otros autores. }\end{array}$ & 1 & 2 & 13 \\
\hline 4 & $\begin{array}{l}\text { Identificar la tesis y las ideas centrales es } \\
\text { definitivo para hacer una crítica seria y } \\
\text { profunda. }\end{array}$ & & 1 & 15 \\
\hline 5 & $\begin{array}{l}\text { Confrontar con otros autores el tema leído } \\
\text { facilita la emisión de juicios críticos sobre su } \\
\text { contenido. }\end{array}$ & 1 & 2 & 13 \\
\hline 6 & $\begin{array}{l}\text { La ficha contribuye a respetar los derechos } \\
\text { de autor. }\end{array}$ & 1 & 1 & 14 \\
\hline 7 & $\begin{array}{l}\text { La ficha contribuye a identificar } \\
\text { contradicciones, valores e ideologías } \\
\text { subyacentes en el texto. }\end{array}$ & & 2 & 14 \\
\hline 8 & $\begin{array}{l}\text { La ficha facilita la comprensión del texto } \\
\text { leído. }\end{array}$ & & & 16 \\
\hline 9 & $\begin{array}{l}\text { Descubrir bondades o amenazas en los } \\
\text { planteamientos del autor del texto facilita la } \\
\text { postura crítica ante ellas. }\end{array}$ & 1 & & 15 \\
\hline 10 & $\begin{array}{l}\text { En términos generales la ficha propicia } \\
\text { lectura crítica. }\end{array}$ & & & 16 \\
\hline \multicolumn{2}{|c|}{ TOTAL } & 4 & 8 & 148 \\
\hline
\end{tabular}

Fuente: Elaboración propia a partir de esta investigación. 


\section{DisCuSIÓN}

Impulsar la lectura crítica en el aula equivale a espolear al estudiante hacia el estudio activo, consciente y deliberado; a tener una experiencia significativa de lectura que lo lleve no solo a interpretar, analizar y reflexionar aquello que lee, sino a leer para ser mejor persona, profesional y ciudadano. Según Girón, Jiménez y Lizcano (2008),

La lectura tiene un lugar fundamental en la institución universitaria, porque es una actividad a través de la cual se ha gestado buena parte del pensamiento letrado sobre "el Hombre y el mundo", y porque ha sido un medio fundamental para la construcción del conocimiento y la producción de la tecnología (p. 33).

En consecuencia, el acto lector no puede limitarse a ser un mero mecanismo transmisor de cultura e información, sino a una dimensión del ser humano que debe cultivarse; solo así será posible dar lugar a una generación de egresados capaces de leer y de interpretar las realidades y necesidades humanas, y de promover modos de vida fundados en la reflexión y la evaluación permanente de todo aquello que acontece en su vivir cotidiano.

La alta inconsistencia en lectura comprensiva y crítica detectada en todos los niveles de escolaridad ha desatado una perentoria preocupación por parte del Estado y del sistema educativo en general. Académicamente hablando, la lectura es la actividad formativa por excelencia; en palabras de Miguel de Zubiría Samper (citado por Acosta, 2009), la lectura constituye una puerta privilegiada por la cual ingresan la mayor parte de los conocimientos, razón por la cual viene bien pensar, desde la práctica pedagógica, escenarios promotores de lectura significativa y significante para los estudiantes.

Llegado a este punto, conviene hacer un pequeño paréntesis para decir que la lectura crítica depende directamente de la capacidad comprensiva de las realidades. Esta actividad humana es condición esencial y punto de partida para el lector crítico. Quien hace lectura comprensiva, además de concentrase en captar lo que el texto está exponiendo, debe al mismo tiempo indagar, cuestionar y criticar aquello que lee. Así, por ejemplo, cuando se trata de un texto escrito, hay que ir más allá de lo que los grafemas y fonemas expresan a simple vista; hay que procurar sustraer las filosofías y creencias soterradas en el texto, captar los errores e ilusiones epistémicas, develar aquellas cosas que el autor no vio o no dijo, entre otras. Se trata entonces de poner en tela de juicio todo lo que el texto enmarca y de someterlo a un discernimiento racional con el fin de extraer su savia y evitar que se pierda o se deseche ingenuamente. 
En este sentido, la lectura comprensiva ayuda a desentrañar los sentidos y significados subyacentes en los textos y discursos (Rumelhart en Serrano, 2007).

Si bien la comprensión lectora alude, sobre todo, a la capacidad para aprehender los puntos álgidos planteados por un autor, en captar los significados transmitidos a través de grafemas, fonemas, imágenes, movimientos, etc.; la lectura crítica, por su parte, constituye un acto lector pormenorizado, activo y deliberado. Se trata de «un esfuerzo de conciencia y una postura comprometida, lúcida y transformadora respecto del acto lector» (Girón, Jiménez y Lizcano, 2008, p. 10). El pensamiento crítico, por su parte, centra sus esfuerzos en reflexionar y evaluar la consistencia y veracidad de los razonamientos presentes en los textos; imprime un carácter epistémico a la actividad lectoral en tanto implica analizar y evaluar de manera exhaustiva la información, la validez de lo leído y decidir deliberadamente qué aceptar o rechazar.

Esta brevísima digresión permite inferir que la lectura crítica se encuentra entre las fronteras de la lectura comprensiva y el pensamiento crítico. Paradójicamente hablando, no es ni lo uno ni lo otro, pero existe, habita y demanda de estos dos componentes cognitivos. La comprensión lectora, como ya se declaró, evoca a una lectura detallada, activa, analítica y reflexiva que requiere de la activación de ciertas habilidades cognitivas y ciertos recursos didácticos para poder desempeñarla satisfactoriamente. El pensamiento crítico, por su parte, responde a una actitud y a una manera particular de pensar y afrontar la vida, la historia y el mundo; se trata de una competencia desarrollada con el tiempo y la práctica. No obstante, según Serrano (2007):

El debido despliegue de la capacidad comprensiva abre espacios de reflexión que permiten repensar y renovar los procesos formativos para elevar la calidad, en la cual la comprensión critica forma parte fundamental en la formación de la persona, dotándola de una clara conciencia y capacidad para ejercer sus derechos civiles y democráticos. (p. 58)

El panorama anterior hace entrever la importancia que tienen los procesos de comprensión de textos y la lectura crítica en todos los seres humanos y especialmente en los estudiantes universitarios, que de una u otra manera se enfrentan diariamente a un texto escrito, y que muchas veces lo hacen sin ninguna técnica o estrategia que les permita altos niveles de comprensión. Por esta razón, el docente debe contemplar en su práctica, instrumentos que posibiliten diferenciar información, organizarla, construir relaciones, construir representaciones, levantar significados y construirlos (Coll, 1991). Dichos mecanismos tienen la facultad de generar elementos estratégicos para comprender y construir conocimiento (Cassany, 2004). Noddings (1995) 
declara en su libro Filosofía de la educación, un suceso un tanto sorprendente y a la vez muy cotidiano, manifiesta que tanto los filósofos como los educadores coinciden en el mutuo interés por la promoción del pensamiento crítico, sin embargo, no han logrado conciliar respecto de en qué consiste y mucho menos en cómo enseńarlo. Esta paradoja refleja la realidad que padece el sistema educativo, y particularmente un buen número de profesores que se limitan a teorizar y pregonar discursos que reclaman la urgencia de ciudadanos y profesionales críticos, pero que en el aula no encuentran la manera de concretizar este ideal.

El uso intencional de mecanismos y dispositivos conducentes a propiciar lectura comprensiva, reflexiva y crítica en el ámbito escolar no solo recaen en el estudiante, sino que es un compromiso del profesor. En tal sentido, es necesario que el educador adapte y proponga una gama de estrategias y recursos impulsadores del sujeto lector activo y crítico. En esta acometida, es necesario que el docente, al diseñar estrategias didácticas de lectura, incorpore en ellas el espíritu innovador e investigativo, a fin de promover en sus estudiantes lectura de orden superior. En el actual panorama histórico, no basta ser usuario pasivo de la información, se requiere superar la idea de leer para subsistir y dar paso al lector activo, capaz de analizar, interpretar y proponer mundos posibles en favor de sí mismos y de la humanidad. Para tal efecto, el hábito lector y la ejercitación en esta actividad intelectual debe ser una constante en el aula y fuera de ella.

\section{Conclusiones}

A pesar de que en ningún momento del proceso investigativo se conceptualizó, ni se dio referente teórico alguno sobre la lectura crítica, los estudiantes describen el sentido y significado de esta expresión, la cual concuerda con la versión de autores de reconocimiento nacional e internacional. Estas son algunas conceptualizaciones realizadas por los estudiantes con relación a la lectura crítica: «Capacidad humana para evaluar los contenidos planteados por los autores», "proceso mediante el cual se hace análisis detallado de un texto y se interpreta la información presente en él», «ejercicio de análisis realizado para descubrir las ideas fundamentales de un texto y dar un veredicto personal sobre ellas», etc. Estas concepciones encajan con las posturas de los eruditos, quienes comprenden la lectura crítica como un tipo de lectura profunda que va más allá de acopiar información literal y se adentra en los terrenos del análisis, la comprensión y la crítica fundamentada (Serrano, 2007; Girón, Jiménez y Lizcano, 2008; Pérez, 2010). 
Los grandes teóricos de la educación sugieren la necesidad de implementar estrategias didácticas, orientadas a la consecución de la promoción de la lectura de nivel superior. En este sentido, la triangulación de los diarios de clase permitió evidenciar que la ficha de lectura crítica, como estrategia pedagógica, dio lugar a que los estudiantes adoptaran una actitud más comprometida y madura frente al acto lector; hizo comprender a los estudiantes participantes acerca de la estructura textual y de cómo los textos se construyen en torno a ideas fundamentales, sin las cuales los documentos pasarían a ser solo una aglomeración de ideas sueltas.

Si bien los estudiantes de manera generalizada encuentran en la ficha de lectura crítica una potencial herramienta que auspicia la lectura con profundidad y a mejorar el nivel académico, en la práctica, un considerable número de jóvenes no pasan de plantear posturas personales, desprovistas de argumentos válidos y coherentes. Sigue vigente una fuerte brecha entre teoría y práctica, en este caso a desplegar lectura de nivel crítico.

Los principales percances suscitados durante la actividad investigativa fueron la dificultad a la hora de identificar y sintetizar el tema central por parte de los estudiantes; la resistencia a no expresar la macroestructura textual o las macroproposiciones con las propias palabras, porque, generalmente, no son valoradas por el profesor que las solicita. Desde esta situación, se puede inferir que la calificación y aprobación por parte del docente constituyen dos factores que impiden a los estudiantes explorar otras formas de lectura distintas a la literal. Acontece, entonces, un suceso paradójico y contradictorio a la vez. Ciertas prácticas pedagógicas pueden terminar por inhibir y castrar el pensamiento autónomo y crítico en los educandos.

Asimismo, se presentó dificultad al momento de reconstruir y redactar las macroproposiciones semánticas sustraídas de los textos; ello ha sido un obstáculo enorme, especialmente, cuando, de manera verbal, los educandos evidencian haber captado la estructura semántica fundamental, pero a la hora de plasmarla gramaticalmente por escrito, esta se pierde o se describe parcialmente.

\section{RECOMENDACIONES}

Si bien la ficha de lectura crítica, en esta investigación, ha sido implementada para encaminar sigilosamente al estudiante hacia una auténtica experiencia de lectura comprensiva y crítica, de tal manera que este se vea abocado no solo a escuchar el texto desde la perspectiva del autor, sino desde él como interprete 
y evaluador, también puede ser aprovechada para otros propósitos educativos. En investigación, por ejemplo, puede ayudar en el proceso de revisión bibliográfica y documental, en la cual se consignen autores y teorías que responden a los objetivos previstos. De igual manera, puede ser utilizada como estrategia de sistematización y análisis de información.

La ficha de lectura ha sido pensada, desde su concepción, en un formato estándar con el ánimo de que pueda disponerse no solo para la lectura de textos argumentativos-descriptivos, como en el caso de la investigación que nos ocupa, sino para cualquier realidad factible de ser leída, interpretada, analizada. A ello responde, precisamente, el apartado de la ficha en el cual se solicita establecer el tipo de texto, objeto de lectura.

Por otra parte, la ficha de lectura crítica representa un importante recurso para formar en el respeto a la voz del otro. En este sentido, antes que propiciar costosas campańas contra el plagio y los derechos de propiedad intelectual, se puede optar por facilitar al estudiante mecanismo que le ayuden a valorar, reconocer y referenciar la producción de los otros. Los componentes zona de identificación y campo de referenciación bibliográfico, presentes en la ficha de lectura crítica, tienen esta misión.

Por lo demás, se espera que esta acción pedagógico-didáctica avizore un devenir promisorio para la vida académica de los estudiantes y, en un futuro no lejano, para la sociedad en general, la cual se beneficiará con ciudadanos y profesionales capaces de leer con criticidad la problemática vigente y plantear alternativas de solución dignas, oportunas y eficaces. El equipo investigador espera contribuir con los resultados de esta investigación a los propósitos de la educación en general y de la práctica pedagógica en particular, pero, de manera especial a la comunidad de estudiantes, que tengan la posibilidad de adueñarse de sus propios procesos de aprendizaje y demás saberes requeridos para generar una sana interacción social y un servicio profesional idóneo y humano.

Sin embargo, conviene decir que la lectura crítica no está supeditada a ninguna fórmula, método o dispositivo en particular. Si bien los recursos mediáticos son útiles y necesarios como mecanismo de activación, entrenamiento y promoción, e inciden rotundamente en la conquista de la dimensión crítica de lectura, esta es y será siempre un asunto de actitud y compromiso del ser humano frente a esta actividad cotidiana. 


\section{REFERENCIAS BIBLIOGRÁFICAS}

Acosta, I. (2009). La comprensión lectora, enfoques y estrategias utilizadas durante el proceso de aprendizaje del idioma español como segunda lengua (Tesis doctoral). Granada: Única.

Caballero, E. (2008). Comprensión lectora de los textos argumentativos en los niños de poblaciones vulnerables escolarizados en quinto grado educación básica primaria (Tesis de Maestría en Educación con Énfasis en Didáctica de la Lectoescritura en la Infancia). Universidad de Antioquia, Medellín.

Calderón, A. y Quijano, J. (2010) Características de comprensión lectora en estudiantes universitarios, Revista Estudios Socio-Jurídicos, 12(1), 337-364.

Cassany, D. (2004). Explorando las necesidades actuales de comprender. Aproximaciones a la comprensión crítica. Lectura y Vida, 25(2), 6-23.

Coll, C. (1991). Psicología y currículo. Barcelona: Paidós.

Creswell, J. (2009). Research Design Qualitative, Quantitative, and Mixed Methods Approaches. Los Angeles, CA: Sage.

García, P. (2011). Bases para la comprensión organizativa del texto. Revista de Lingüistica y Lenguas Aplicadas, 6, 125-137.

Girón, S., Jiménez, C. y Lizcano, C. (2008). ¿Cómo hacer lectura crítica? Colección Cuadernillo Serie Gramática. Bogotá: Universidad Sergio Arboleda.

Lahuerta, A. (1996). El uso de la estructura textual como instrumento metodológico para mejorar la comprensión lectora de estudiantes de inglés como lengua extranjera. Aula Abierta, 67, 193-216.

Matéus, G. (2007). Psicología de la comprensión textual y control de la comprensión. Revista Folios, 26, 39-48.

McNamara, D. (2004). Aprender del texto: efectos de la estructura textual y las estrategias del lector. Revista Signos, 37(55), 19-30. https://doi. org/10.4067/S0718-09342004005500002

Noddings, N. (1995). Philosophy of education. Tercera edición. Colorado: Westview Press.

Ochoa, S. y Aragón, L. (2005). Comprensión lectora y funcionamiento metacognitivo en estudiantes universitarios. Universitas Psychologica, 4(2), 179-196. Bogotá: Pontificia Universidad Javeriana.

Pérez, A. (2010). La lectura crítica en los libros de texto de educación secundaria (Trabajo final del máster en Lingüística y aplicaciones tecnológicas Especialidad en Aprendizaje de Lenguas). Universitat Pompeu Fabra, Barcelona.

Rugarcía, A. (1999). Los valores y las valoraciones en la educación. México: Trillas. 
Serrano, S. (2007). Competencias de lectura crítica. Una propuesta para la reflexión y la práctica. Universidad de los Andes. Facultad de humanidades y educación. Revista Acción Pedagógica, 16(enero-diciembre), 58-68.

Tarrés, M., Montenegro, S., D’Ottavio, A. y García, E. (2008). La lectura crítica del artículo científico como estrategia para el aprendizaje del proceso de investigación. Revista Iberoamericana de Educación, 45(6), 229-237.

Taylor, S. J. y Bogdan, R. (1986). Introducción: ir hacia la gente. En Introducción a los métodos cualitativos de investigación. México: Paidós.

Taylor, S. J. y Bogdan, R. (2000). Introducción a los métodos cualitativos de investigación. Tercera edición. México: Paidós.

Villarroel, R. (2010). Conocimiento metacognitivo y comprensión lectora. Investigación profesoral. Chile: Universidad de los Lagos.

Zárate, A. (2010). La lectura crítica en los libros de texto de educación secundaria. Concepción y tratamiento metodológico. Barcelona: Universidad de Pompeu Fabra, Departamento de Traducción y Ciencias del Lenguaje. 